\title{
Quantitative ellipsometric microscopy at the silicon-air interface
}

\author{
F. Linke and R. Merkel ${ }^{a)}$ \\ Institut für Schichten und Grenzfä̈chen, Institut 4: Biologische Schichten (ISG4), Forschungszentrum Jülich \\ GmbH, D-52425 Jülich, Germany
}

(Received 8 December 2004; accepted 3 April 2005; published online 17 May 2005)

\begin{abstract}
Ellipsometric microscopy is a technique that combines the merits of ellipsometry and light microscopy, i.e., it allows noninvasive, label-free measurements of thin film thickness and refractive index at high lateral resolution. Here we give a detailed description of the technique including a complete calibration scheme and a model to correct for the instrumental polarization of the imaging optics. The performance of the instrument was studied experimentally. We found a lateral resolution of $1 \mu \mathrm{m}$ and an absolute height accuracy of $3 \mathrm{~nm}$. The measured refractive indices were accurate to $2.3 \%$ and the height sensitivity of the instrument was smaller than $5 \AA$. Another virtue of the instrument design besides its good performance is that it is in essence an extension of standard light microscopy and could be integrated into commercial microscopes.
\end{abstract}

(C) 2005 American Institute of Physics. [DOI: 10.1063/1.1921547]

\section{INTRODUCTION}

Ellipsometry is a versatile optical technique for the investigation of thin films $(0.1-300 \mathrm{~nm})$. Film thickness and refractive index can be obtained rapidly and with high precision. However, most available instruments exhibit an area of measurement of $\sim 1 \mathrm{~mm}^{2}$. Therefore microsctructured surfaces cannot be studied with these instruments. In the past several schemes of imaging ellipsometers have been proposed and some instruments are already commercially available. Traditional approaches of imaging ellipsometry frequently utilize one of the following alternatives: either the surface is scanned by a focused laser beam ${ }^{1,2}$ or the sample is illuminated by parallel light and the surface imaged obliquely onto a charge coupled device (CCD) camera. ${ }^{3-7}$ Scanning the sample is time consuming. Therefore most efforts concentrated on the latter approach. Its advantage is the ease of attaching the necessary imaging optics to existing point ellipsometers. However, due to the tilt angle of the objective its numerical aperture is limited, resulting in a lateral resolution of $\approx 3-5 \mu \mathrm{m}$. Because of oblique imaging the image plane is inclined, too, causing image distortion. At high magnifications the limited depth of focus gives rise to clear image formation only in a narrow strip. ${ }^{8}$

In ellipsometric microscopy an optical microscope is modified for ellipsometric measurements. Most of the above mentioned limitations of microellipsometers were thus overcome. ${ }^{9}$ Recently, first spatially resolved ellipsometric measurements at the glass-water interface were reported. ${ }^{10}$ While these studies showed that the principle of ellipsometric microscopy indeed works, the accuracy and reliability of the ellipsometric data left much space for improvement. Thus the next step in the development of ellipsometric microscopy was an in depth characterization of the instrument performance combined with an error analysis. This is the

\footnotetext{
${ }^{a)}$ Author to whom correspondence should be addressed; electronic mail: r.merkel@fz-juelich.de
}

focus of the present article. Here we present a complete calibration procedure, describe the crucial systematic error sources, and show how to correct for these errors. Most important was to account for the influence of the imaging optics on the polarization of light. Finally, we present actual measurements of the lateral resolution of the ellipsometric microscope. In this work we concentrated on the technically important air-silicon interface.

\section{ROTATING-ANALYZER ELLIPSOMETRY}

In reflection ellipsometry a sample is illuminated with parallel, monochromatic light of well defined polarization. The measured quantity is the change of polarization due to reflection of light at the sample. Using the Fresnel equations for the propagation of light in layered media ${ }^{11}$ optical properties (refractive index $n$, thickness $d$ ) of layers thinner than the wavelength of the light itself can be inferred. Usually, the change in polarization is described by the ellipsometric angles $\Psi$ and $\Delta$ which are defined by the ratio of the complex reflection coefficients:

$$
\tan \Psi \cdot e^{i \Delta}=\frac{R_{p}}{R_{s}}=\frac{\left|R_{p}\right|}{\left|R_{s}\right|} \cdot e^{i\left(\varphi_{p}-\varphi_{s}\right)} .
$$

The design of the ellipsometric microscope was based on a rotating-analyzer type ellipsometer. ${ }^{12}$ In this type of ellipsometer the incoming light is linearly polarized at an angle $P$ with respect to the plane of incidence. Light reflected at the sample is analyzed by traversing a rotating polarizer, the analyzer. The primary measured quantity in this instrument design is the intensity of the light that passes the analyzer set at an azimuthal angle $A, I(A)$. This intensity can be computed from the electrical field strength at the detector $\mathbf{E}_{\text {Det }}$.

$$
\mathbf{E}_{\text {Det }}=\mathbf{R}_{-A} \mathbf{T}_{\mathbf{A}} \mathbf{R}_{A} \mathbf{T}_{\mathbf{S}} \mathbf{R}_{-P}\left(\begin{array}{c}
E_{0} \\
0
\end{array}\right)
$$

with 


$$
\mathbf{T}_{\mathrm{A}}=\left(\begin{array}{ll}
1 & 0 \\
0 & 0
\end{array}\right), \quad \mathbf{T}_{\mathrm{S}}=\left(\begin{array}{cc}
R_{p} & 0 \\
0 & R_{s}
\end{array}\right)
$$

representing Jones matrices ${ }^{11}$ for the analyzer $\left(\mathbf{T}_{\mathbf{A}}\right)$ and the specimen $\left(\mathbf{T}_{\mathbf{S}}\right)$. $\mathbf{R}_{X}$ represents the rotation matrix (with rotation angle $X$ )

$$
\mathbf{R}_{X}=\left(\begin{array}{cc}
\cos X & \sin X \\
-\sin X & \cos X
\end{array}\right)
$$

$A$ and $P$ denote the azimuthal angles of the polarizer and the analyzer with respect to the plane of incidence of the sample. $E_{0}$ represents the electric field strength transmitted by the polarizer. Exploiting Eq. (2) one finds for the measured intensity $I(A)$ at the detector

$$
I(A)=\mathbf{E}_{\text {Det }}^{\dagger} \cdot \mathbf{E}_{\text {Det }}=I_{0}[1+a \cos (2 A)+b \sin (2 A)]
$$

where

$$
\begin{aligned}
& I_{0}=\frac{E_{0}^{2}}{2}\left(R_{p} R_{p}^{*} \cos ^{2} P+R_{s} R_{s}^{*} \sin ^{2} P\right), \\
& a=\frac{R_{p} R_{p}^{*} \cos ^{2} P-R_{s} R_{s}^{*} \sin ^{2} P}{R_{p} R_{p}^{*} \cos ^{2} P+R_{s} R_{s}^{*} \sin ^{2} P}, \\
& b=\frac{2 \Re\left(R_{p} R_{s}^{*}\right) \cos P \sin P}{R_{p} R_{p}^{*} \cos ^{2} P+R_{s} R_{s}^{*} \sin ^{2} P} .
\end{aligned}
$$

The symbol $\mathfrak{R}(z)$ denotes the real part of the complex number $z$. The definition of the ellipsometric angles [Eq. (1)] together with Eqs. (6) and (7) yields

$$
\begin{aligned}
& \tan \Psi=|\tan P| \cdot \sqrt{\frac{1+a}{1-a}}, \\
& \cos \Delta=\operatorname{sgn}(\cos P \sin P) \cdot \frac{b}{\sqrt{1-a^{2}}} .
\end{aligned}
$$

The function $\operatorname{sgn}(x)$ returns the sign of $x$. Because the polarizer orientation $P$ is known it is possible to determine $\Psi$ and $\Delta$ of the sample by measuring the Fourier coefficients $a, b$ of the normalized intensity $I(A) / I_{0}$ incident at the detector. The remaining quadrant ambiguity of $\Delta$ can be resolved by a second measurement with an inserted $\lambda / 4$ wave plate (the compensator). Because the phase shift of the compensator just adds to the phase shift $\Delta$ introduced by the sample effectively $\cos \left(\Delta+90^{\circ}\right)=-\sin \Delta$ is measured. This second measurement resolves the quadrant ambiguity. Thus, a typical measurement yields two sets of ellipsometric quantities: a set of quadrant corrected $(\Psi, \Delta)$ from the measurement without compensator and a second set of quadrant corrected $\left(\Psi^{c}, \Delta^{c}\right)$ from the measurement with compensator.

By fitting a model function to measured $\Psi^{(c)}$ and $\Delta^{(c)}$ values one can extract up to two optical quantities of the layers constituting the sample. This model function is usually calculated by applying the Fresnel formulas, valid for stratified, planar layers. ${ }^{11}$

\section{EXPERIMENTAL SETUP}

The setup was constructed as an inverted microscope employing epi-illumination (cf. Fig. 1): A parallel, polarized

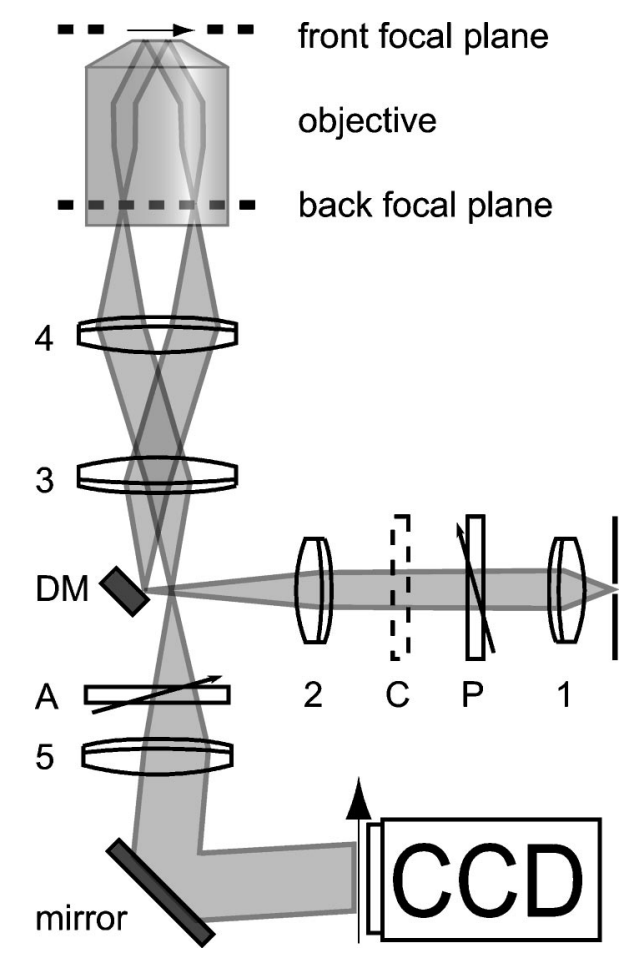

FIG. 1. Design of the ellipsometric microscope: lenses 2-4 focused a parallel beam of light into an off-axis point of the back focal plane, resulting in oblique illumination of the object in the front focal plane. The incoming light passed a polarizer $(P)$. The angle of incidence in the front focal plane was controlled by horizontally shifting the rigidly coupled system of lens 2 and deflection mirror (DM). An optional compensator $(C)$ allowed illumination with elliptically polarized light. Light reflected and diffracted at the object was collected by the objective, passed a computer controlled analyzer $(A)$ and was focused onto a CCD camera (lens 5). Lenses 3 and 4 formed a telescopic system, thus they influenced the magnification of the microscope. Here the illumination beam path is depicted, i.e., wherever a focus is indicated, the illumination pinhole was imaged. Lens 5 imaged the pinhole to infinity and formed an image of the object on the CCD at the same time.

beam of light was focused into the back focal plane of an infinity-corrected high-power microscope objective (MPlanApo $50 \times$, numerical aperture $=0.95$, Olympus). The parallel beam of light was created by spatially filtering monochromatic light $(546.1 \mathrm{~nm})$ from a mercury arc lamp (HBO 103, Osram) with a small pinhole $(50 \mu \mathrm{m})$ and imaging it to infinity (lens 1). The finite diameter of the pinhole introduced a small divergence of the beam $\left(\approx \pm 0.03^{\circ}\right)$. The beam traversed a polarizer (Glan-Thompson of interferometric quality: PGT2.12+PGT0.1, B. Halle, Berlin). A crucial point in the design was that the angle of incidence could be controlled in order to maximize ellipsometric contrast. By laterally displacing the mechanically connected system of deflection mirror and lens 2 the pinhole was imaged (lenses 3 and 4) into the back focal plane of the objective with a certain displacement to the optical axis. This displacement caused oblique illumination of the object plane.

At the object light was reflected and diffracted. This light was collected by the objective and imaged onto a CCD camera (C4880-50, Hamamatsu, Herrsching). The infinity space between the tube lens (lens 5) and objective accommodated the optical components necessary for both illumination of the object and polarization analysis of the reflected light. As the image is maximally blurred in this region those components 
created almost no image degradation. For each pixel the intensity $I(A)$ was measured as a function of the analyzer angle $A$. Thus, each pixel of the CCD acted as a stand-alone ellipsometer in a rotating-analyzer configuration.

As commercially available microscopes did not offer enough space for the necessary modifications the setup was realized on an optical bench. All lenses were high grade achromatic doublets (Melles Griot) with antireflection coatings optimized for the wavelength used. Dust particles on the optical components were troublesome, because at the low numerical aperture of illumination used here they caused highly visible diffraction rings. The abundance of these artifacts was greatly reduced by encapsulating all optical components in a tube mounting system (Linos, Göttingen).

The polarizers were mounted in stepper motor driven goniometers (DRT 65, Owis, Staufen). A compensator (0th order $\lambda / 4$ plate, Owis) mounted in a motorized filter wheel (Filterwheel 40, Owis) could be rotated into the optical path. All these devices were computer controlled. Ellipsometric measurements were performed by acquiring two stacks of pictures (with and without compensator), each taken typically at 18 equally spaced analyzer angles between $0^{\circ}$ and $170^{\circ}$. Background images, where the illuminating light bundle was blocked by a shutter, were recorded with the same exposure time (typically $80-110 \mathrm{~ms}$ for silicon substrates) as used for the actual measurements and subtracted from the measured data in order to correct for dark current and amplification offsets of the CCD. A measurement cycle (acquisition of 72 images) typically lasted for $4 \mathrm{~min}$. Most of this time was spent to adjust the rotating components. At the moment the compensator is rotated in and out at each analyzer setting which is very time consuming. By streamlining the measurement sequence the measurement time can be reduced to about $90 \mathrm{~s}$. This time could be further cut in half if the background images recorded at the first measurement were reused for subsequent measurements.

For processing of raw data a separate image processing software was developed that computed the ellipsometric angles $\Psi$ and $\Delta$ for each pixel of the camera (typically $30 \mathrm{~s}$ on a $2.5 \mathrm{GHz}$ Pentium compatible). The inherent quadrant ambiguity of $\Delta$ was automatically resolved by combining the two data sets with and without compensator. Within the software it was possible to define a model for the optical properties of the planar stratified layers of the sample and to fit the corresponding model function to either whole pictures one-dimensional (1D) fit of $1024^{2}$ pixels: $15 \mathrm{~min}, 2 \mathrm{D}$ fit: $3 \mathrm{~h}$ ) or selected profiles of $\Psi$ and $\Delta$ (1D fit: $<1$ s, 2D fit: $<10 \mathrm{~s}$ ). By this procedure refractive index $n$ and thickness $d$ of layers were determined with high spatial resolution.

\section{SAMPLE PREPARATION}

The samples used for calibration and measurements were thin structured films of $\mathrm{MgF}_{2} \quad(n=1.389)$ (99.99\%, Goodfellow, Bad Nauheim) and gold deposited upon a silicon (100) substrate by vacuum deposition. Thin films of $\mathrm{ZnS}$ $(n=2.379)$ were also deposited and measured to check the performance of the ellipsometric microscope in a completely different refractive index regime. Silicon wavers (Wacker
Siltronic, Burghausen) were taken as substrates. The silicon wavers were cleaned by successive ultrasonification in pure de-ionized water produced by a Millipore apparatus (Milli-Q Reagent Grade Water System, $R>18 \mathrm{M} \Omega \mathrm{cm}^{-1}, p \mathrm{H} 5.5$, Millipore, Molsheim, France), ethanol and acetone for $15 \mathrm{~min}$, each. Between each sonification step the wavers were rinsed ten times with de-ionized water. Microstructures were obtained by using an electron microscopy grid as mask during the deposition (thermal evaporation, BOC Edwards Auto 306 Turbo, Kirchheim: substrate temperature $25^{\circ} \mathrm{C}$, pressure $2 \times 10^{-6} \mathrm{mbar}$, deposition rate: $4-7 \mathrm{~nm} / \mathrm{s}$ ). The optical properties of thin films depend on the deposition parameters and must be determined experimentally. To this end a second mask allowed for deposition within a $6 \times 8 \mathrm{~mm}^{2}$ region in close vicinity to the structured region. On these macroscopic plateaus refractive index and layer thickness were measured with high accuracy using a commercial point ellipsometer $(\lambda=632.8 \mathrm{~nm}$, Plasmos $\mathrm{GmbH}$, München). The measured refractive indices were wavelength corrected to $546.1 \mathrm{~nm}$ by multiplying with ratios

$$
\frac{n(\lambda=546.1 \mathrm{~nm})}{n(\lambda=632.8 \mathrm{~nm})}= \begin{cases}1.0012 & \text { for } \mathrm{MgF}_{2} \\ 1.0145 & \text { for } \mathrm{ZnS}\end{cases}
$$

extracted from the literature. ${ }^{13-15}$

\section{CALIBRATION}

Calibration of the setup described above involved two different issues: the incident angle of the light in the object plane had to be known depending on the displacement position of the deflection mirror. Second, all azimuthal angles of the polarizers and the compensator had to be adjusted precisely with respect to the plane of incidence.

\section{A. Illuminating incident angle}

Aplanatic systems obey Abbe's sine condition ${ }^{16,17}$ which implies that the paraxial approximation of principal planes is replaced by a construction with principal spheres. ${ }^{17}$ From Abbe's sine condition the relation between incident angle $\Theta$ and displacement of the deflection mirror $d_{M}$ was calculated as

$$
\sin \Theta=\frac{d_{M}}{n \cdot f_{\mathrm{obj}}} \cdot \frac{f_{4}}{f_{3}},
$$

where $n$ denotes the refractive index of the object side medium (here air, $n=1.000$ ) and $f_{\text {obj }}$ the focal length of the objective. The magnification due to lenses 3 and 4 was explicitly included. Figure 2 shows that the geometrically measured incident angles were in excellent agreement with Eq. (10). Similar results were found earlier for an oil immersion objective. ${ }^{10}$ Therefore, in the following all given incident angles $\Theta$ were computed from the instrument settings with the help of Eq. (10).

\section{B. Azimuthal angles of the polarizing components}

The azimuth angles $P, A$, and $C$ of the rotating components of our setup had to be calibrated with respect to the plane of incidence of the sample. To this end we adapted two established methods from rotating-analyzer ellipsometry. 


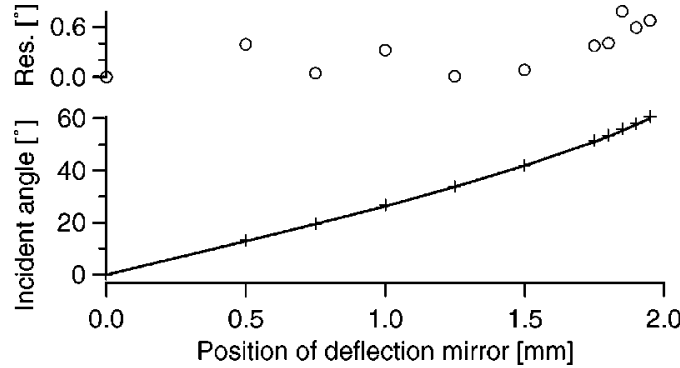

FIG. 2. The incident angle in the object plane in dependence of the distance of the deflection mirror to the optical axis. Geometrically measured angles $\Theta$ (crosses) are in excellent agreement with the predictions from Abbe's sine condition (line) with $f_{\mathrm{obj}}=3.6 \mathrm{~mm}$ (the straight line is not a fit).

One of these methods (Aspnes) ${ }^{18}$ yields excellent results for $\Delta$ values far away from $0^{\circ}$ and $180^{\circ}$ while the other one $(\mathrm{de} N \mathrm{Nijs})^{19}$ performs best in the complementary range of $\Delta$ values.

According to Aspnes the polarizer azimuth reading $\delta P$ corresponding to the plane of incidence can be found by measuring the residual function $R(P)$ at several polarizer settings $P$ :

$$
R(P)=1-\eta^{-2}\left(a^{2}+b^{2}\right) .
$$

The instrumental frequency attenuation factor $\eta$ describes the attenuation of the $2 \omega$ frequency components $\left(a \cdot I_{0}, b \cdot I_{0}\right)$ with respect to the dc component $I_{0}$ due to electrical circuit deficiencies. A first order expansion in $P=P^{\prime}-\delta P$ for $P \approx 0$ yields

$$
R\left(P^{\prime}\right) \approx\left(1-\frac{1}{\eta^{2}}\right)+\frac{4 \sin ^{2} \Delta}{\eta^{2} \tan ^{2} \Psi}\left(P^{\prime}-\delta P\right)^{2}+\ldots
$$

and for $P \approx \pi / 2$

$$
\begin{aligned}
R\left(P^{\prime}\right) \approx & \left(1-\frac{1}{\eta^{2}}\right)+\frac{4 \sin ^{2} \Delta \tan ^{2} \Psi}{\eta^{2}}\left(P^{\prime}-\delta P-\frac{\pi}{2}\right)^{2} \\
& +\ldots,
\end{aligned}
$$

i.e., in both zones the unknown polarizer offset $\delta P$ can be found by fitting a parabola to the measured residual function $R(P)$. Because the curvature of this function becomes very low for samples with $\Delta$ close to $0^{\circ}$ or $180^{\circ}$ this method cannot be applied to such samples. In these cases the method of de Nijs was applied. In this approach the phase difference function $\Phi\left(P^{\prime}\right)$

$$
\Phi\left(P^{\prime}\right)=\theta\left(P^{\prime}\right)-\theta\left(P^{\prime}+\pi / 2\right),
$$

where $\theta\left(P^{\prime}\right)$ is the phase function

$$
\theta\left(P^{\prime}\right)=\arctan (b / a) / 2
$$

has to be measured at several $P^{\prime}$ settings. A first order expansion of $\Phi\left(P^{\prime}\right)$ yields

$$
\Phi\left(P^{\prime}\right) \approx \frac{2 \cos \Delta}{\tan 2 \Psi} \cdot\left(P^{\prime}-\delta P\right),
$$

i.e., $\delta P$ can be found by determining the $x$ axis intercept of a straight line fitted to the measured data.

Determination of the analyzer azimuth offset $\delta A$ is identical for both methods: once the polarizer offset $\delta P$ was found $\delta A$ can be extracted from the phase function $\theta\left(P^{\prime}\right)$ measured at $P^{\prime}=\delta P$. This can be seen from the first order expansion of $\theta\left(P^{\prime}\right)$ in both $A$ and $P$ :

$$
\begin{aligned}
\theta\left(P^{\prime}\right) \approx & \delta A+\frac{\cos \Delta}{\tan \Psi} \cdot\left(P^{\prime}-\delta P\right)+\ldots(P \approx 0), \\
\theta\left(P^{\prime}\right) \approx & \delta A+\cos \Delta \tan \Psi \cdot\left(P^{\prime}-\delta P-\frac{\pi}{2}\right) \\
& +\ldots(P \approx \pi / 2) .
\end{aligned}
$$

It was possible to apply the methods described above to ellipsometric microscopy by measuring laterally homogeneous samples at several polarizer settings $P$ and computing the Fourier coefficients $a$ and $b$ from laterally averaged intensities $(\sim 25 \times 25 \mu \mathrm{m})$.

Table I and Fig. 3 summarize the results of a typical calibration. Three different samples were chosen: a bare gold substrate (500 nm gold deposited onto a silicon waver) optimally suited for residual function calibration, a bare silicon substrate optimal for the phase difference calibration, and a silicon substrate covered with $40 \mathrm{~nm} \mathrm{MgF}_{2}$ which allows for both calibrations. It turned out that the polarizer offsets $\delta P_{1}$ and $\delta P_{2}$ found in different $P$ zones $P \approx 0$ or $\pi / 2$ differed up to $0.6^{\circ}$. Consequently the analyzer offsets $\delta A_{1}$ and $\delta A_{2}$ differed too. This discrepancy was explained by residual ellipticity $\Gamma_{P, A}$ of the utilized polarizing components. This imperfection affects both calibration procedures described above. ${ }^{20}$ If $\delta P_{1}$ and $\delta A_{1}$ represent experimentally determined azimuthal correction values found near $P \approx 0^{\circ}$ in the residual function calibration method, those values have to be corrected by

$$
\begin{aligned}
& \delta P_{c}=\delta P_{1}-\frac{\Gamma_{A} \tan \Psi^{\prime}+\Gamma_{P} \cos \Delta^{\prime}}{\sin \Delta^{\prime}}, \\
& \delta A_{c}=\delta A_{1}-\frac{\Gamma_{P} \cot \Psi^{\prime}+\Gamma_{A} \cos \Delta^{\prime}}{\sin \Delta^{\prime}},
\end{aligned}
$$

where $\Psi^{\prime}$ and $\Delta^{\prime}$ denote zero order approximations (without residual ellipticity corrections). ${ }^{18,20}$ The effect of the residual ellipticity on the polarizer calibration angles retrieved from the phase difference function method is given to first order by

$$
\begin{aligned}
& \delta P_{c}=\delta P_{0}+\Gamma_{P} \frac{\tan \Delta^{\prime}}{\cos 2 \Psi^{\prime}}, \\
& \delta A_{c}=\delta A_{0}+\Gamma_{P} \frac{\sin \Delta^{\prime}}{\tan 2 \Psi^{\prime}} .
\end{aligned}
$$

Here $\delta P_{0}$ is the $P^{\prime}$ axis intercept of the phase difference function and $\delta A_{0}$ is the phase function evaluated at $\delta P_{0}$. According to Ref. 18 the residual ellipticities $\Gamma_{P, A}$ can be calculated from measured $\delta P_{1}, \delta P_{2}, \delta A_{1}$, and $\delta A_{2}$ values by the following equations:

$$
\Gamma_{P}=\left[\left(\delta P_{1}-\delta P_{2}\right) \cos \Delta^{\prime}-\frac{\delta A_{1}-\delta A_{2}}{\sin 2 \Psi^{\prime}}\right] / D_{1},
$$


TABLE I. Calibration results for three different samples: $40 \mathrm{~nm} \mathrm{MgF}$ on a silicon substrate, a bare gold substrate and a bare silicon substrate. $\Psi$ and $\Delta$ values were computed from the curvature and slope of the residual and phase function. These values are still affected by the instrumental polarization (cf. Sec. VI B). $\delta P_{1,2}$ and $\delta A_{1,2}$ were determined from the residual function measurements at $P^{\prime} \approx 0^{\circ}, P^{\prime} \approx 90^{\circ}$, respectively. $\delta P_{0}$ and $\delta A_{0}$ were obtained from measuring the phase difference function. Entries "- " indicate that the respective calibration method were not applicable in this case. The residual ellipticities $\Gamma_{P}$ and $\Gamma_{A}$ were determined by Eqs. (23)-(25). For the bare silicon sample the average of the residual ellipticities $\Gamma_{P, A}$ as determined on gold and on $\mathrm{MgF}_{2}$ were used. Using those values the azimuthal angle offsets were corrected according to the respective calibration procedure [Eqs. (19) and (20) and Eqs. (21) and (22), respectively]. This yielded $\delta P_{c}$ and $\delta A_{c}$.

\begin{tabular}{|c|c|c|c|c|}
\hline Method & Quantity & $\mathrm{MgF}_{2}$ & $\begin{array}{l}\text { Sample } \\
\text { gold }\end{array}$ & Silicon \\
\hline Residual function & $\eta_{1}$ & 1.0024 & 1.004 & 1.0018 \\
\hline Residual function & $\eta_{2}$ & 0.9908 & 0.994 & 0.9913 \\
\hline Residual function & $\delta P_{1}\left(^{\circ}\right)$ & 0.354 & 0.267 & 一 \\
\hline Residual function & $\delta P_{2}\left(^{\circ}\right)$ & 0.919 & 0.511 & - \\
\hline Residual function & $\delta A_{1}\left(^{\circ}\right)$ & -0.214 & 0.015 & - \\
\hline Residual function & $\delta A_{2}\left({ }^{\circ}\right)$ & -0.247 & 0.010 & 一 \\
\hline Phase difference function & $\delta P_{0}\left(^{\circ}\right)$ & -0.035 & - & 0.024 \\
\hline Phase difference function & $\delta A_{0}\left({ }^{\circ}\right)$ & 0.249 & - & 0.164 \\
\hline Residual function & $\Gamma_{P}\left[\cdot 10^{-3}\right]$ & -4.4 & -1.5 & - \\
\hline \multirow[t]{3}{*}{ Residual function } & $\Gamma_{A}\left[\cdot 10^{-3}\right]$ & -6.0 & -2.6 & 一 \\
\hline & $\Psi\left({ }^{\circ}\right)$ & 34.4 & 44.1 & 31.9 \\
\hline & $\Delta\left({ }^{\circ}\right)$ & 144.5 & 126.8 & 173.0 \\
\hline Residual function & corr. $\delta P_{c}\left(^{\circ}\right)$ & 0.41 & 0.38 & 一 \\
\hline Phase difference function & corr. $\delta P_{c}\left(^{\circ}\right)$ & 0.46 & - & 0.11 \\
\hline Residual function & corr. $\delta A_{c}\left({ }^{\circ}\right)$ & -0.06 & 0.02 & - \\
\hline Phase difference function & corr. $\delta A_{c}\left(^{\circ}\right)$ & -0.13 & - & 0.09 \\
\hline
\end{tabular}

$$
\Gamma_{A}=\left[\left(\delta A_{1}-\delta A_{2}\right) \cos \Delta^{\prime}-\frac{\delta P_{1}-\delta P_{2}}{\sin 2 \Psi^{\prime}}\right] / D_{1},
$$

$$
D_{1}=2\left(\cos ^{2} \Delta^{\prime}-\sin ^{-2} 2 \Psi^{\prime}\right) / \sin \Delta^{\prime} .
$$

Table I shows that the polarizer offsets $\delta P_{c}$ and $\delta A_{c}$ found after correcting for the influence of the residual ellipticities $\Gamma_{P, A}$ were in good agreement for all three samples. In this calibration average values of $P_{c}=0.34^{\circ} \pm 0.16^{\circ}$ and $A_{c}$ $=-0.02^{\circ} \pm 0.10^{\circ}$ were found. In order to estimate the sensitivity of this calibration method on the range of angles used the analysis was repeated utilizing only the subrange $P$ $\in\left[-5^{\circ}, 5^{\circ}\right]$. The agreement was very good $\left(P_{c}\right.$ $=0.35^{\circ} \pm 0.18^{\circ}$ and $\left.A_{c}=-0.05^{\circ} \pm 0.13^{\circ}\right)$ and showed that the calibration procedures were indeed very stable.

In the setup the azimuthal angle of the compensator was not automated. Therefore it was impossible to apply likewise calibration procedures. The principal axes of the compensator were found by placing the already calibrated polarizer (oriented at $P=45^{\circ}$ ), compensator, analyzer, and detector in line and adjusting the azimuthal angle of the compensator in order to minimize variations in $I(A)$. In the manually found minimum position the orientation of the axes of the compensator should enclose an angle of $45^{\circ}$ with the transmission axis of the polarizer. This kind of calibration procedure minimizes $|\delta P-\delta C|$. Therefore the azimuthal errors with respect to the plane of incidence of the sample should satisfy $\delta C$ $\approx \delta P \approx 0^{\circ}$ when all components are reassembled to the ellipsometric microscope.

\section{MEASUREMENTS AND DISCUSSION}

In order to assess the accuracy of the ellipsometric microscope, homogeneous areas of the $\mathrm{MgF}_{2}$ coated silicon substrates were measured. All presented ellipsometric quantities were computed from laterally averaged $(\sim 25$ $\times 25 \mu \mathrm{m})$ intensities. However, averaging could be omitted as well, (cf. Sec. VI D). Additionally, the presented values were zone averaged in order to avoid systematic errors like component azimuth errors $(\delta P, \delta A$, and $\delta C)$ and polarizer imperfections $\Gamma_{A, P}$ : those errors are eliminated to first order if measured $\Psi$ and $\Delta$ values, obtained at polarizer setting $P$ and $-P$, are averaged. This procedure is called zone averaging. ${ }^{20,21}$

Great care was taken to accurately characterize the deposited thin films with a commercial point ellipsometer. The measured film thicknesses and the $\lambda$-corrected refractive indices were used to compute theoretically expected ellipsometric quantities $\Psi$ and $\Delta$. Figure 4 shows the results in comparison to these theoretical data. While qualitatively the agreement between measured and expected data was remarkably good, systematic disagreements were clearly visible. Even more disturbing was the fact that measurements with and without compensator showed considerable disagreement. Furthermore it was impossible to compute valid $\Delta$ values at several thicknesses: the experimentally determined Fourier coefficients $a$ and $b$ produced right-hand sides of Eq. (9) with moduli larger than one. In Fig. 4 the corresponding values were set to an arbitrarily selected value of $70^{\circ}$. 


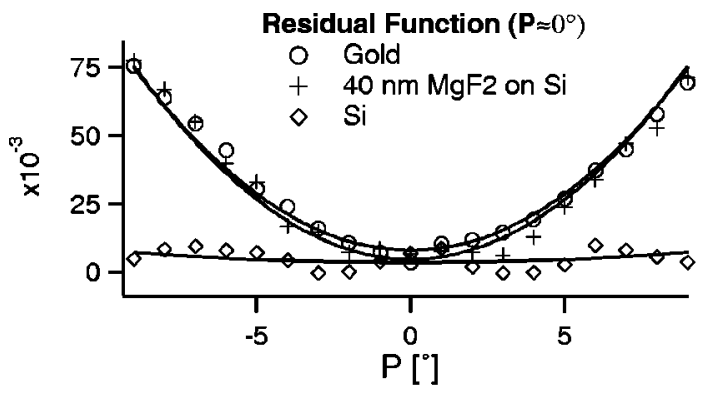

(a)

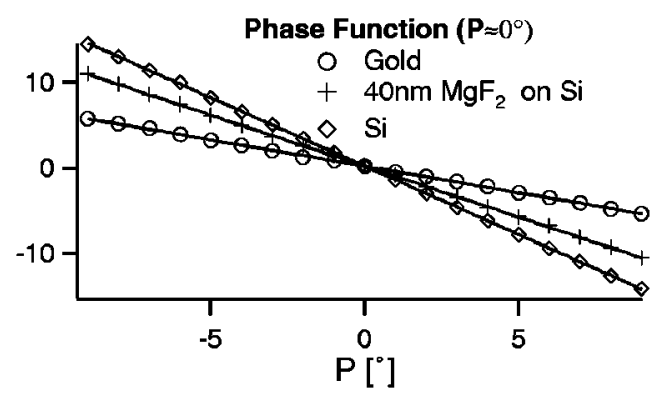

(c)

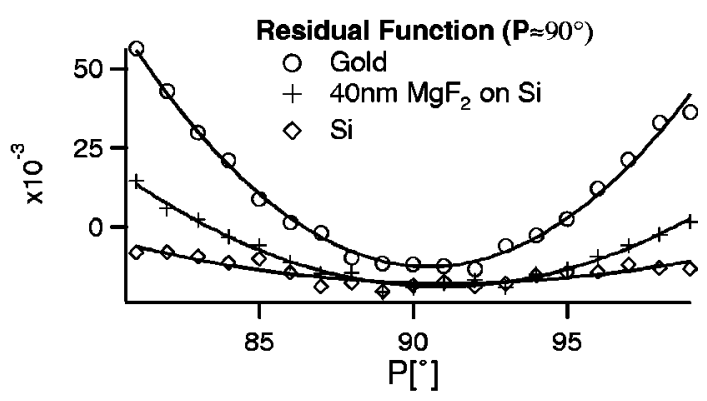

(b)

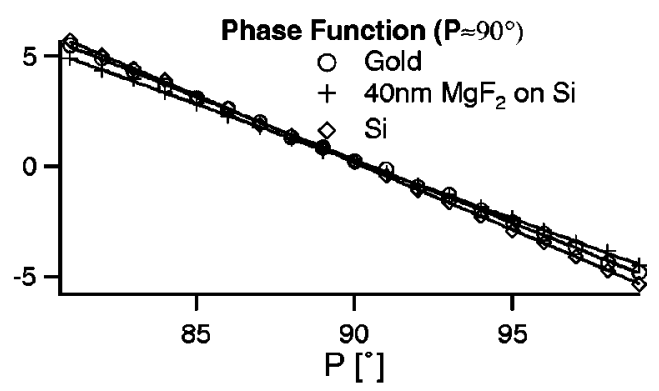

(d)

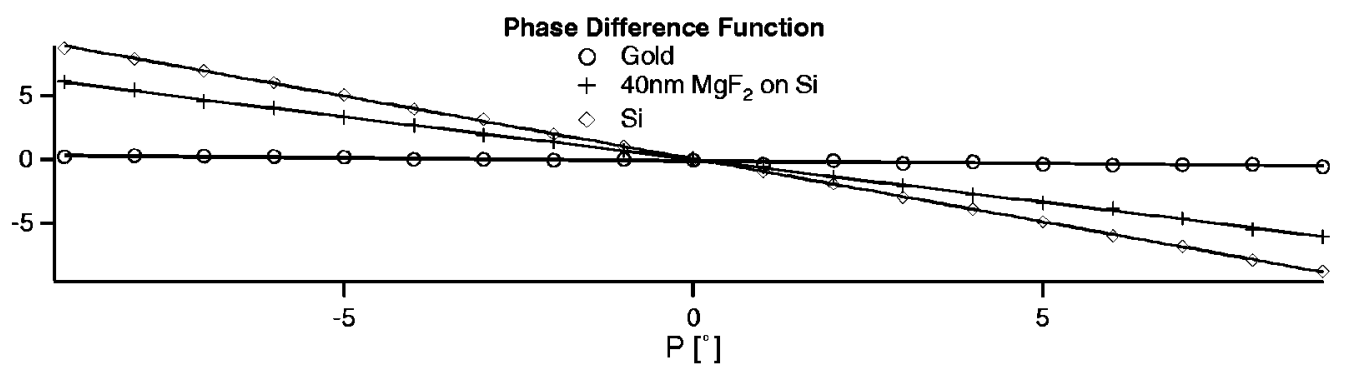

(e)

FIG. 3. Plot of the residual (a), (b), the phase (c), (d), and the phase difference function (e) as measured with the ellipsometric microscope for three different samples: gold substrate, $40 \mathrm{~nm} \mathrm{MgF}_{2}$ on silicon and a bare silicon substrate.

\section{A. Detector nonlinearity}

The reason for the latter two issues turned out to be a slight nonlinearity of the utilized CCD camera. In contrast to nonlinearity, blooming (i.e., smearing of charge into neighboring pixels) and image persistence did not influence our results. We tested the linearity of the CCD camera with two different methods: the intensities incident on the CCD camera were varied by inserting neutral density filters and by varying the acquisition time of the CCD camera. Both methods yielded similar (slightly nonlinear) results. This nonlinearity may be dealt with by two different approaches: either the measured intensities are linearized directly by generating a lookup table of the inverse of the measured linearity (interpolated with cubic splines), or the nonlinearity is corrected for at the level of the measured Fourier coefficients $a$ and $b$. The latter can be done by assuming a small quadratic nonlinearity of the detector output $I \sim I+k I^{2}$ and computing the relation between true and apparent Fourier coefficients. Correcting the nonlinearity at the level of the Fourier coefficients turned out to yield results of similar quality, but did not succeed in correcting uncomputable ellipsometric quantities as frequently. Thus, the nonlinearity was always corrected at the intensity level. This correction brought data with and without a compensator into good agreement (Fig. 4), nevertheless a considerable difference to the theoretical expected data remained.

\section{B. Instrumental polarization}

This remaining discrepancy was found to stem from instrumental polarization, ${ }^{22}$ i.e., the influence of the imaging optical components on the state of polarization. There are two major causes for instrumental polarization: birefringence of the optical components induced by stress due to mounting the components and the nonnormal incidence on the optical surfaces as the light passes through the imaging system. Instrumental polarization is difficult to treat from first principles. Therefore we developed a phenomenological, well motivated, and simple approach to account for it. Although correcting for instrumental polarization is essential for accurate measurements such an approach has not been published before.

Our model of the instrumental polarization treats the imaging optics formally in exactly the same way as the sample 

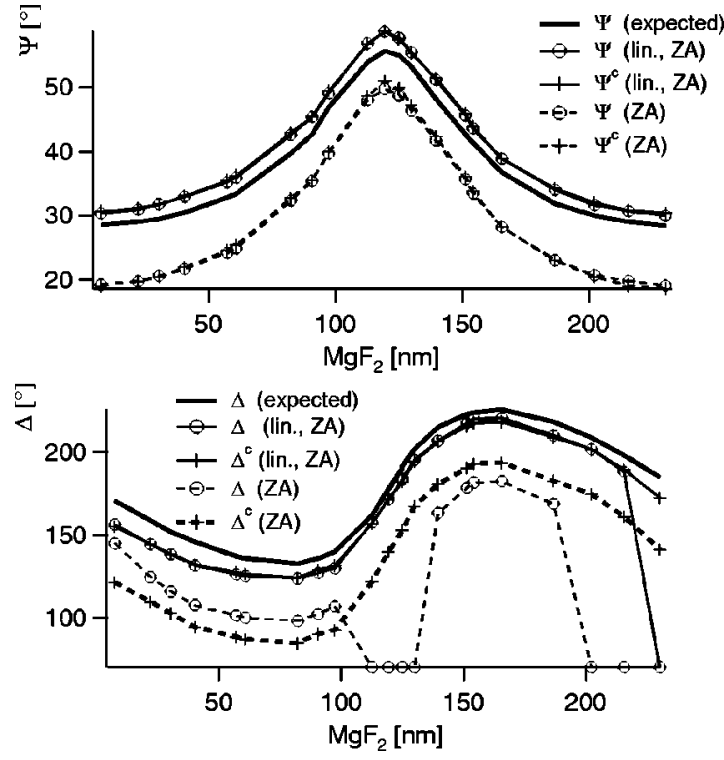

FIG. 4. Measured ellipsometric quantities in comparison to expected data (thick lines): Shown are plain and uncorrected $\Psi$ and $\Delta$ values obtained at an incident angle $\Theta=55.3^{\circ}$ from $\mathrm{MgF}_{2}$ coated silicon substrates [broken lines, for clarity the data was shifted by $-10^{\circ}(\Psi)$ and $-30^{\circ}(\Delta)$, respectively]. Measurements with (crosses) and without (circles) compensator show considerable disagreements in $\Delta$. Correcting for the slight nonlinearity of the CCD camera removed this discrepancy (thin lines) and rendered most former uncomputable $\Delta$ values (arbitrarily set to $70^{\circ}$ ) to sensible data. Nevertheless a considerable difference to the theoretical expected data remained. This was due to the instrumental polarization introduced by the imaging optics.

itself: its influence is parameterized with values $\Psi_{\mathrm{Obj}}$ and $\Delta_{\text {Obj }}$. As we illuminate and observe the sample through the objective the Jones matrix of the sample

$$
\left(\begin{array}{cc}
\tan \Psi e^{i \Delta} & 0 \\
0 & 1
\end{array}\right)
$$

in Eq. (2) is then replaced by

$$
\left(\begin{array}{cc}
\tan \Psi_{\mathrm{Obj}} e^{i \Delta_{\mathrm{Obj}}} & 0 \\
0 & 1
\end{array}\right) \cdot\left(\begin{array}{cc}
\tan \Psi e^{i \Delta} & 0 \\
0 & 1
\end{array}\right) \cdot\left(\begin{array}{cc}
\tan \Psi_{\mathrm{Obj}} e^{i \Delta_{\mathrm{Obj}}} & 0 \\
0 & 1
\end{array}\right) .
$$

In this model the ellipsometric measurements $\Psi^{\prime}$ and $\Delta^{\prime}$ of the combined system "imaging system+object" can be corrected for the influence of the imaging system with the help of the following equations:

$$
\begin{aligned}
& \tan \Psi^{\prime}=\tan \Psi \cdot \tan ^{2} \Psi_{\mathrm{Obj}}, \\
& \Delta^{\prime}=\Delta+2 \Delta_{\mathrm{Obj}},
\end{aligned}
$$

if the parameters $\Psi_{\mathrm{Obj}}$ and $\Delta_{\mathrm{Obj}}$ of the imaging system are known. We determined those parameters by minimizing the sum

$\sum_{i}\left\{\left[\Psi_{i}^{T}-\tan ^{-1}\left(\frac{\tan \Psi_{i}^{\prime}}{\tan ^{2} \Psi_{\mathrm{Obj}}}\right)\right]^{2}+\left[\Delta_{i}^{T}-\left(\Delta_{i}^{\prime}-2 \cdot \Delta_{\mathrm{Obj}}\right)\right]^{2}\right\}$,

where $\Psi_{i}^{T}$ and $\Delta_{i}^{T}$ are the theoretically expected values and $\Psi_{i}^{\prime}$ and $\Delta_{i}^{\prime}$ are the experimentally determined (nonlinearity corrected and zone averaged) ellipsometric quantities of Fig. 4. The index $i$ comprises all performed measurements, including the ones with an inserted compensator. For the em-
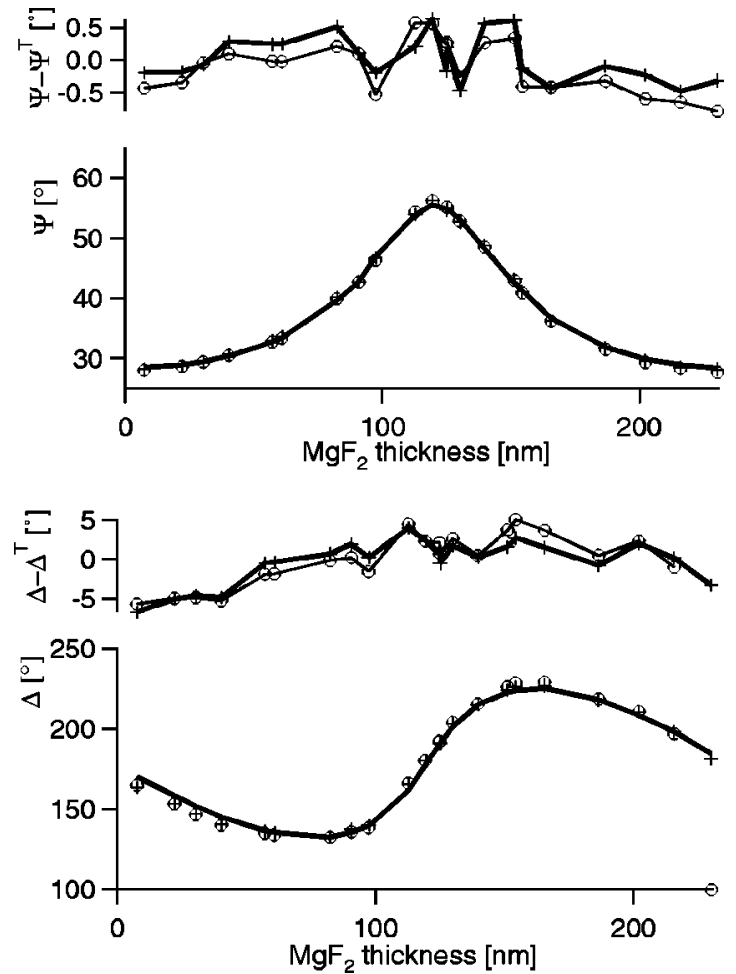

FIG. 5. Linearized and instrumental polarization corrected ellipsometric quantities (thin lines) in comparison to expected data (thick line). The angle of incidence was $\Theta=55.31^{\circ}$. Data with (crosses) and without compensator (circles) are in good agreement and show similar residuals.

ployed objective the following values were found: $\Psi_{\text {Obj }}$ $=46.33^{\circ}$ and $\Delta_{\mathrm{Obj}}=-4.51^{\circ}$. Figure 5 shows the result of this correction procedure.

In order to check the validity of the found calibration scheme (detector nonlinearity+instrumental polarization) several tests were made. The questions addressed by these tests were: (i) is the calibration scheme valid in the complete domain of the ellipsometric quantities $\left(\Psi \in\left[0^{\circ}, 90^{\circ}\right], \Delta\right.$ $\in\left[0^{\circ}, 360^{\circ}[)\right.$ ?), (ii) does it hold for incident polarizations $P \neq 45^{\circ}$ (as theoretically expected)?, and (iii) do the quantities $\Psi_{\mathrm{Obj}}$ and $\Delta_{\mathrm{Obj}}$ vary with the incident angle $\Theta$ ?

To address the first question silicon substrates were covered with thin films of $\mathrm{ZnS}(n=2.38)$. These samples test exactly the complement of the $\Psi$ domain already measured with the $\mathrm{MgF}_{2}$ coated samples and their $\Delta$ values cover almost the whole domain $\left[0^{\circ}, 360^{\circ}\right]$. The latter two points were tested by varying the polarizer setting angle $P$ and the incident angle. The values found for $\Psi_{\mathrm{Obj}}$ and $\Delta_{\mathrm{Obj}}$ were in excellent agreement with the values found utilizing the $\mathrm{MgF}_{2}$

TABLE II. Experimentally determined instrumental depolarization parameters of the imaging components.

\begin{tabular}{cccc}
\hline \hline Material & Parameters & $\Psi_{\mathrm{Obj}}\left(^{\circ}\right)$ & $\Delta_{\mathrm{Obj}}\left({ }^{\circ}\right)$ \\
\hline $\mathrm{MgF}_{2}$ & $\Theta=55.31^{\circ}, P=45^{\circ}$ & 46.33 & -4.51 \\
$\mathrm{ZnS}$ & $\Theta=53.13^{\circ}, P=45^{\circ}$ & 46.60 & -4.54 \\
$\mathrm{ZnS}$ & $\Theta=55.31^{\circ}, P=45^{\circ}$ & 46.58 & -4.44 \\
$\mathrm{ZnS}$ & $\Theta=53.13^{\circ}, P=30^{\circ}$ & 46.73 & -4.36 \\
$\mathrm{ZnS}$ & $\Theta=55.31^{\circ}, P=30^{\circ}$ & 46.59 & -4.53 \\
\hline \hline
\end{tabular}




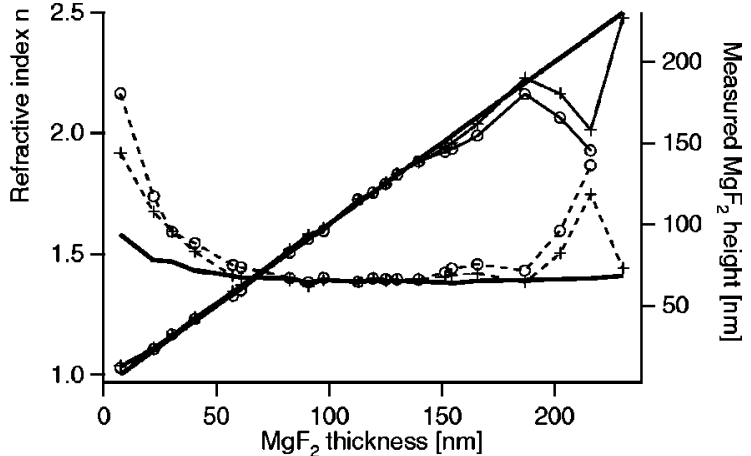

(a)

2D-t
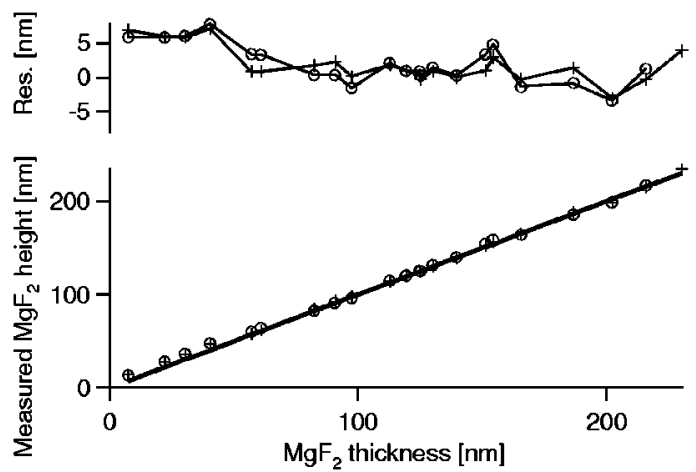

(b)

1D-t
FIG. 6. (a) Refractive indices (broken lines) and thicknesses (thin lines) obtained simultaneously by means of a $2 \mathrm{D}$ fit from corrected $\Psi$ and $\Delta$ : Shown are data measured with (crosses) and without (circles) compensator. The thick line represents expected data determined with a commercial point ellipsometer. (b) Resulting thicknesses if the refractive index of $\mathrm{MgF}_{2}$ $(n=1.389)$ is imposed.

samples. The results of these tests are summarized in Table II.

\section{Accuracy}

Having ensured that the ellipsometric microscope measures $\Psi$ and $\Delta$ accurately the next step was to evaluate its accuracy with respect to determination of the optical properties of thin films. Figure 6(a) shows the results obtained by fitting $n$ and $d$ simultaneously to the corrected ellipsometric data. While for intermediate thicknesses the results were in excellent agreement with the expected values, the results scattered considerably at very small layer thicknesses. The same was true for layer thicknesses close to the period of the ellipsometric quantities. This behavior is a well known limitation of ellipsometry: near to layer thicknesses of
TABLE III. Accuracy of the ellipsometric microscope when the refractive index is imposed. Measurements with $\left(\overline{\left|\Delta d^{c}\right|}\right)$ and without $(\overline{|\Delta d|})$ compensator show nearly the same accuracy.

\begin{tabular}{cccc}
\hline \hline Material & Parameters & $\mid \overline{|\Delta d|}(\mathrm{nm})$ & $\overline{\left|\Delta d^{c}\right|}(\mathrm{nm})$ \\
\hline $\mathrm{MgF}_{2}$ & $\Theta=55.31^{\circ}, P=45^{\circ}$ & 2.6 & 2.2 \\
$\mathrm{ZnS}$ & $\Theta=53.13^{\circ}, P=45^{\circ}$ & 2.4 & 3.0 \\
$\mathrm{ZnS}$ & $\Theta=55.31^{\circ}, P=45^{\circ}$ & 1.4 & 1.7 \\
$\mathrm{ZnS}$ & $\Theta=53.13^{\circ}, P=30^{\circ}$ & 2.6 & 2.7 \\
$\mathrm{ZnS}$ & $\Theta=55.31^{\circ}, P=30^{\circ}$ & 1.4 & 1.5 \\
\hline
\end{tabular}

$d \approx k \cdot D_{\Theta}\left\{k \in\{0,1,2, \ldots\}, D_{\Theta}=\lambda / 2 n_{j}\left[1-\left(1 / n_{j}^{2}\right) \sin ^{2} \Theta\right]^{-1 / 2}\right\}$ the ellipsometric quantities become insensitive to the refractive index $n_{j}$ of the layer. Hence the retrieved refractive indices became erroneous. As in every interferometric technique the optical thickness $n \cdot d$ was still measured accurately and the determined thickness was erroneous too. In these cases one optical quantity has to be imposed in order to extract the other with a high degree of accuracy. Figure 6(b) shows the results of a one dimensional fit where the refractive index was fixed. The obtained thicknesses $d_{i}^{M}$ were in excellent agreement with the expected values $d_{i}$ and the residues were usually well below $5 \mathrm{~nm}$. Table III summarizes the average absolute deviation

$$
\overline{|\Delta d|}=\frac{1}{N} \sum_{i=1}^{N}\left|d_{i}^{M}-d_{i}\right|
$$

of the measured thicknesses from their expected value at several instrument settings. It shows very impressively that the overall height accuracy of the ellipsometric microscope was better than $3 \mathrm{~nm}$ if the refractive index of the thin layer was known. The results of the same analysis for $2 \mathrm{D}$ fits and intermediate thicknesses are summarized in Table IV. Refractive indices were measured to an accuracy of better than $2.5 \%$ while thicknesses were accurate to approximately $2.5 \mathrm{~nm}$.

\section{Vertical sensitivity}

The results presented so far were based on laterally averaged intensities. However, thicknesses could also be measured accurately at every single pixel. Figure 7 shows a fullframe 3D plot of the height topology obtained from two measurements of a $\mathrm{MgF}_{2}$ sample at polarizer azimuths $P=$ $+45^{\circ}$ and $P=-45^{\circ}$. For retrieval of zone averaged and instrumental polarization corrected $\Psi$ and $\Delta$ pictures the recorded intensities were first corrected for the CCD nonlinearity. Afterwards $\Psi^{\prime}$ and $\Delta^{\prime}$ images were computed for both polarizer settings. These images were zone averaged for each

TABLE IV. Accuracy of the ellipsometric microscope when refractive index and thickness are obtained simultaneously.

\begin{tabular}{|c|c|c|c|c|c|}
\hline Material $[$ Range $(\mathrm{nm})]$ & $\Theta, P$ & $\overline{|\Delta d|}(\mathrm{nm})$ & $\overline{\left|\Delta d^{c}\right|}(\mathrm{nm})$ & $\overline{|\Delta n|}$ & $\overline{\left|\Delta n^{c}\right|}$ \\
\hline $\mathrm{MgF}_{2}(50-200)$ & $55.31^{\circ}, 45^{\circ}$ & 2.8 & 2.0 & 0.023 & 0.010 \\
\hline $\mathrm{ZnS}(20-100)$ & $53.13^{\circ}, 45^{\circ}$ & 2.1 & 1.5 & 0.037 & 0.062 \\
\hline $\mathrm{ZnS}(20-100)$ & $55.31^{\circ}, 45^{\circ}$ & 2.1 & 2.4 & 0.040 & 0.056 \\
\hline $\mathrm{ZnS}(20-100)$ & $53.13^{\circ}, 30^{\circ}$ & 2.6 & 2.4 & 0.063 & 0.077 \\
\hline $\mathrm{ZnS}(20-100)$ & $55.31^{\circ}, 30^{\circ}$ & 2.2 & 2.5 & 0.049 & 0.061 \\
\hline
\end{tabular}




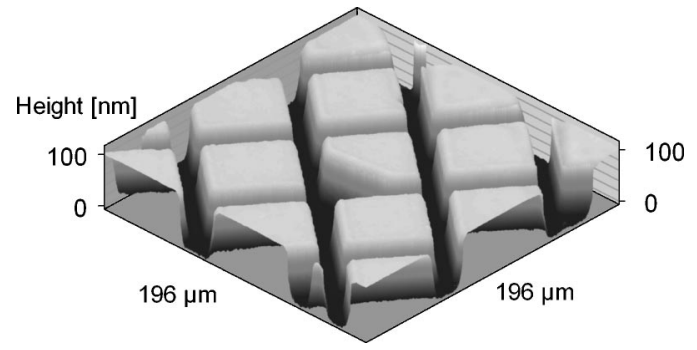

FIG. 7. 3D height topology of a $\mathrm{MgF}_{2}$ sample as obtained by ellipsometric microscopy. The incident angle was $55.31^{\circ}$. At every single pixel of the CCD camera $\left(1024^{2}\right)$ sensible values were obtained. Histograms of heights obtained on homogeneous regions are shown in Fig. 8.

pixel. The resulting $\Psi^{\prime}$ and $\Delta^{\prime}$ pictures were then corrected for instrumental polarization. No other processing was applied. From these $\Psi$ and $\Delta$ pictures the corresponding thickness map was obtained by means of a pixel wise 1D fit with $75 \mathrm{~nm}$ height as the starting value.

Figure 8 shows histograms of the thicknesses obtained pixel wise on laterally homogeneous areas $(\sim 20 \times 10 \mu \mathrm{m})$. A Gaussian fit to the histograms yielded a measure for the standard deviation (s.d.) of the distribution. It was usually extremely narrow (s.d. $\sim 0.5 \mathrm{~nm}$ ). The thick vertical bars in Fig. 8 mark the thicknesses obtained by first averaging laterally the intensities $I(A)$ over the same regions as used for determination of the histograms. The thicknesses obtained by fitting to $\Psi$ and $\Delta$ obtained from laterally averaged intensities should therefore represent the average thickness of that area. The absolute deviation of the results of both procedures was less than $0.5 \mathrm{~nm}$. The small amount of noise present in the obtained heights and the fact that heights determined from laterally averaged intensities did not differ significantly from heights obtained at single pixels proved that laterally averaging was not necessary. Judging from the amplitude of the noise we expect that our apparatus should be able to resolve height steps of less than $0.5 \mathrm{~nm}$.

\section{E. Lateral resolution}

We determined the lateral resolution of the ellipsometric microscope by measuring edge diffraction patterns of microstructures on silicon wavers. These samples were prepared as follows. Thermally oxidized silicon wavers were coated with photoresist which was subsequently microstructured by lithography $(\lambda=365 \mathrm{~nm})$ through a chromium mask specially designed for these samples and written by an electron beam lithograph. After development only regions which were not exposed to ultraviolet light were still covered by resist. A subsequent reactive ion etching process removed the oxide exclusively in regions not covered by resist. In a second reactive ion etching step the remaining resist was removed as well. The final samples exhibited $\mathrm{SiO}_{2}$ structures on bare silicon substrates with an edge width of less than $0.2 \mu \mathrm{m}$ which was determined by scanning electron microscopy.

In order to determine the lateral resolution quantitatively we used the ISO standardized knife-edge method. ${ }^{23}$ This method relies on the measurement of a line profile over a step-like structure. The resulting profile is the outcome of the convolution of the object intensity with the line spread func-

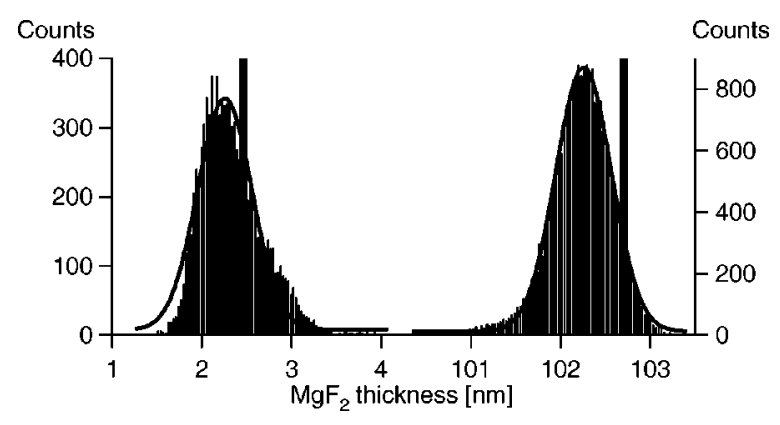

FIG. 8. Histograms of heights - obtained at single pixels - within a rectangular region free of $\mathrm{MgF}_{2}$ (left) and coated with $\mathrm{MgF}_{2}$ (right). A Gaussian fit to the histograms yielded mean heights of $2.25 \pm 0.43 \mathrm{~nm}$ and $102.3 \pm 0.44 \mathrm{~nm}$, respectively. The given uncertainties represent the widths of the height distributions. The thicknesses computed from ellipsometric data which were obtained by laterally averaging the intensities $I(A)$ within the same region are marked with thick vertical bars. The small standard deviations of the histograms prove that there is no need for smoothing the data by lateral averaging.

tion (LSF). By differentiating the measured edge profile numerically one obtains the LSF of the imaging system. The modulation transfer function (MTF) ${ }^{24}$ is then obtained by a Fourier transformation of the LSF

$$
\operatorname{MTF}(k)=|\mathcal{F}(\operatorname{LSF}(x))|=\mathcal{F}\left(\frac{d}{d x^{\prime}} I^{\prime}\left(x^{\prime}\right)\right) .
$$

For the edge structures under consideration this relation can be understood as follows: the MTF is defined as the modulus of the image frequency spectrum normalized to the object frequency spectrum. ${ }^{24}$ Edge structures can be modeled by use of the Heaviside unit function $U(x)$. This yields in agreement with Eq. (29):

$$
\begin{aligned}
\operatorname{MTF}(k) & =\left|\frac{\mathcal{F}\left(I^{\prime}\left(x^{\prime}\right)\right)}{\mathcal{F}(U(x))}\right| \\
& =\left|\frac{\mathcal{F}\left(I^{\prime}\left(x^{\prime}\right)\right)}{1 / i k+\pi \delta(k)}\right| \stackrel{k \neq 0}{=} \mathcal{F}\left(\frac{d}{d x^{\prime}} I^{\prime}\left(x^{\prime}\right)\right) .
\end{aligned}
$$

The MTF is a measure for the contrast still conceivable in the image space depending on the spatial frequency of the object structure. The contrast of the image decreases with the spatial frequency and the frequency where the MTF drops below a value of 0.1 is identical to the one found by the Rayleigh criterion. ${ }^{25}$

Please note that this method is strictly valid only for incoherent imaging systems (e.g. scanners, digital cameras). In ellipsometric microscopy the illumination aperture is extremely small and hence the object is illuminated coherently. Thus image formation can only be described by convolution of the electrical field strengths, but those are inaccessible to direct measurement. Nevertheless Eq. (30) shows that this procedure yields a measure of the maximum transmitted spatial frequency. We used the knife edge method to obtain an objective and reproducible estimate of the contrast of small objects. Because of the coherent superposition of neighboring Airy patterns the observed resolution is dependent on their phase relation. This is why the measured resolution might be dependent on the layer composition of the sample 


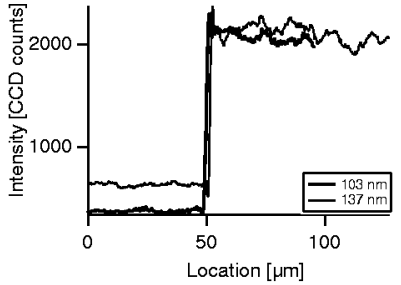

(a)

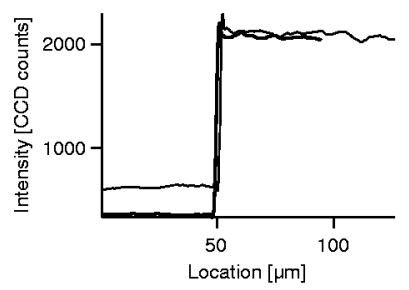

(c)

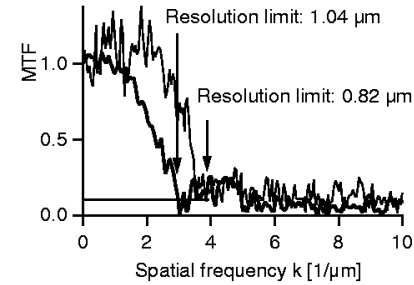

(b)

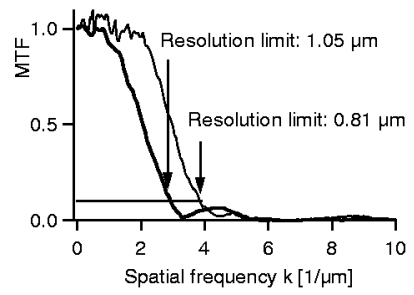

(d)
FIG. 9. (a) shows raw intensity profiles of edge structures (width: 1 pixel, $\Theta=53.13^{\circ}, P=45^{\circ}, A=110^{\circ}$, parallel to the plane of incidence). The resulting MTFs are given in (b). (c) shows the averaged profiles (width: 151 pixels, moving median and average filter with kernel size 5). The resulting MTFs (d) are considerably smoother, but the actual resolution limit did not change.

(material and thicknesses). Therefore we measured samples with different heights and took the maximum value as a measure for the lateral resolution.

For determination of the lateral resolution edge profiles were extracted from three different samples $(25,103$, and $137 \mathrm{~nm} \mathrm{Si}_{2} \mathrm{O}$ height), analyzing intensity, $\Psi, \Delta$, and height profiles. Each of these profiles was extracted from measurements with and without the compensator as well as parallel and perpendicular to the plane of incidence. Altogether 48 profiles were analyzed. Because the sample was illuminated under a certain angle of incidence we expect different lateral resolutions for the various orientations of the structures. In order to measure the diffraction patterns with a high signalto-noise ratio the intensity and $\Psi / \Delta$ profiles were averaged perpendicularly to the profile direction (151 pixels, $29 \mu \mathrm{m}$ ). Height profiles were obtained from the averaged $\Psi / \Delta$ profiles. The strong nonlinear relation between layer thickness and ellipsometric quantities can cause discontinuous height profiles. In order to avoid spurious super-resolution artifacts introduced by these abrupt discontinuities it was necessary to apply averaging filters (moving median and a moving average filter with kernel size 5) to the raw profiles. For reasons of consistency these filters were applied to all profiles, although the much smoother intensity profiles would not demand such measures. As one pixel corresponds to $0.191 \mu \mathrm{m}$ in object space the kernel size 5 corresponds to a length still below the expected resolution. It was verified that for smooth profiles, i.e., intensity and $\Psi / \Delta$ profiles, the applied averaging and filtering procedure did not influence the obtained lateral resolution (cf. Fig. 9).

The results are summarized in Table V. We found that the lateral resolution indeed depends on the spatial direction. For profiles parallel to the plane of incidence we found a lateral resolution of approximately $1 \mu \mathrm{m}$ and for perpendicu-
TABLE V. Conservative estimates of the lateral resolution of the ellipsometric microscope in microns, determined by measurement of its modulation transfer function. Shown are maximum values for the three different sample heights.

\begin{tabular}{lcccc}
\hline \hline \multicolumn{1}{c}{ Direction } & Intensity & Psi & Delta & Height \\
\hline Parallel & 1.1 & 0.8 & 1.1 & 1.1 \\
Parallel (comp.) & 1.1 & 0.8 & 1.4 & 1.4 \\
Perpendicular & 1.7 & 1.3 & 1.4 & 1.5 \\
Perpendicular (comp) & 1.8 & 1.3 & 1.5 & 1.3 \\
\hline \hline
\end{tabular}

lar profiles the resolution was $1.75 \mu \mathrm{m}$. In actual experiments, the sample can always be rotated in order to observe interesting structures with improved resolution.

In summary, our experiments clearly showed that ellipsometric microscopy has matured to a very reliable and accurate technique combining the benefits of light microscopy (high spatial resolution) with those of ellipsometry (good resolution for thickness and refractive index). The most important building block for this progress was correcting for the instrumental polarization of the imaging optics by a simple and reliable procedure.

\section{ACKNOWLEDGMENTS}

The authors are grateful to Professor E. Sackmann and Professor M. Rief for hospitality and continuous support. They also thank C. M. Cesa (Research Center, Julich) for the microstructured silicon wavers. This project was funded partly by the Deutsche Forschungsgemeinschaft (DFG) via Group Grant No. SFB 563.

${ }^{1}$ Q. Zhan and J. Leger, Appl. Opt. 41, 4630 (2002).

${ }^{2}$ M. Erman and J. Theeten, J. Appl. Phys. 69, 859 (1986).

${ }^{3}$ R. Cohn, J. Wagner, and J. Kruger, Appl. Opt. 27, 4664 (1988).

${ }^{4}$ A. Albersdörfer, G. Elender, G. Mathe, K. Neumaier, P. Paduschek, and E. Sackmann, Appl. Phys. Lett. 72, 2930 (1998).

${ }^{5}$ A.-H. Liu, P. Wayner, and J. Plawsky, Appl. Opt. 33, 1223 (1994).

${ }^{6}$ T. Beaglehole, Rev. Sci. Instrum. 59, 2557 (1988).

${ }^{7}$ G. Jin, R. Jansson, and H. Arein, Rev. Sci. Instrum. 67, 2930 (1996).

${ }^{8}$ H. Harke, R. Teppner, O. Schulz, H. Orendi, and H. Motschmann, Rev. Sci. Instrum. 68, 3130 (1997).

${ }^{9}$ K. Neumaier, G. Elender, E. Sackmann, and R. Merkel, Europhys. Lett. 49, 14 (2000).

${ }^{10}$ F. Linke and R. Merkel, IEE Proc., Nanobiotechnol. 151, 95 (2004).

${ }^{11}$ R. Azzam and N. Bashara, Ellipsometry and Polarized Light (North Holland, Amsterdam, 1996).

${ }^{12}$ P. Hauge and F. Dill, IBM J. Res. Dev. 17, 472 (1973).

${ }^{13}$ M. Dodge, Appl. Opt. 23, 1980 (1984).

${ }^{14}$ CrystalTechno LTD, 〈http://www.crystaltechno.com/Materials/ZnS.htm.

${ }^{15}$ Opto-Technological Laboratory, 〈http://www.optotl.ru/ZnSEng.htm.〉

${ }^{16}$ M. Born and E. Wolf, Principles of Optics: Electromagnetic Theory of Propagation, Interference and Diffraction of Light (Cambridge University Press, New York, 1999).

${ }^{17}$ H. Haferkorn, Bewertung Optischer Systeme (Verlag der Wissenschaften, Berlin, 1986).

${ }^{18}$ D. Aspnes, J. Opt. Soc. Am. 64, 812 (1974).

${ }^{19}$ J. d. Nijs, A. Holtslag, and A. v. Silfhout, J. Opt. Soc. Am. A 5, 1466 (1988).

${ }^{20}$ R. Collins, Rev. Sci. Instrum. 61, 2029 (1990).

${ }^{21}$ J. d. Nijs and A. v. Silfhout, J. Opt. Soc. Am. A 5, 773 (1988).

${ }^{22}$ R. Chipman, Opt. Eng. (Bellingham) 28, 90 (1989).

${ }^{23}$ ISO 12233, Photography-Electronic Still-Picture Cameras-Resolution Measurements (International Organization for Standardization, 2000).

${ }^{24}$ M. Pluta, Advanced Light Microscopy, Principles and Basic Properties (Elsevier, New York, 1989), Vol. 1.

${ }^{25}$ D. Williams and P. Burns, \&Proceedings of IST's PICS Conference, 2001. 